\title{
CULTURA DA CESARIANA: FATORES RELACIONADOS A ALTA TAXA DO PROCEDIMENTO NO BRASIL
}

Bruna Soares de Souza Lima Rodrigues, Adriano Lucarelli Margarido, Ana Flávia Salgado Gomes, Ana Karolina Carvalho Andrade, Dayane Batista Ferreira, Isabella Santos Zanetti, Raquel Roque Rodrigues. Cultura da Cesariana: fatores relacionados a alta taxa do procedimento no Brasil. Revista Saúde Dinâmica, vol. 1, núm. 2, 2019. Faculdade Dinâmica do Vale do Piranga.

SAÚDE DINÂMICA - Revista Científica Eletrônica FACULDADE DINÂMICA DO VALE DO PIRANGA

2a Edição 2019 | Ano I-no 2 | ISSN-XXXX-XXXX

2o semestre de 2019 


\title{
Cultura da cesariana: fatores relacionados a alta taxa do procedimento no brasil
}

Cesarian culture: factors related to high rate procedure in brazil

Bruna Soares de Souza Lima Rodrigues ${ }^{I^{*}}$, Adriano Lucarelli Margarido ${ }^{l}$, Ana Flávia Salgado Gomes $^{l}$, Ana Karolina Carvalho Andrade ${ }^{l}$, Dayane Batista Ferreira ${ }^{I}$, Isabella Santos Zanetti ${ }^{I}$, Raquel Roque Rodrigues ${ }^{1}$.

${ }^{1}$ Faculdade Dinâmica do Vale do Piranga, Rua G, 205 - Bairro Paraíso - Ponte Nova - MG - Cep: 35430-302, * brunasoaressl@yahoo.com.br

\section{Resumo}

O Brasil é um dos líderes na realização de cesarianas no mundo, mesmo diante dos riscos do procedimento quando comparado ao parto normal. Nesse sentido, este estudo apresenta como objetivos: analisar os possíveis fatores relacionados a essa escolha, demonstrar o papel da mulher e do médico na tomada de decisão e compilar possíveis alternativas para diminuição do alto índice deste procedimento. Para alcançar os objetivos propostos, foi realizada uma pesquisa bibliográfica nas bases de dados Scielo e Lilacs e foram selecionados 31 artigos, disponíveis online. Após fichamento desses periódicos, foi possível constatar que a alta incidência de cesarianas é resultado, principalmente, da falta de informação, sobre a cirurgia, por parte da gestante e do interesse do profissional obstetra pela realização de tal procedimento. Os trabalhos analisados sugerem, ainda, que ações de incentivo ao parto normal, como: a desmistificação da dor e do sofrimento, a divulgação dos benefícios e a melhor remuneração e assistência pelo acompanhamento do trabalho de parto normal podem reverter o atual quadro brasileiro.

Palavras-chave: cesariana, incidência, gestantes, parto normal.

\begin{abstract}
Brazil is one of the leaders in performing caesarean sections in the world, despite the risks of the procedure when compared to normal parturition. In this sense, this study aims to: analyze the possible factors related to this choice, demonstrate the women and doctors role in decision making and compile possible alternatives to reduce the high rate of this procedure. To achieve the proposed objectives, a bibliographic search was performed in the Scielo and Lilacs databases and 31 available online articles were selected. After recording these journals, it was found that the high incidence of caesarean sections is, mainly, due to the lack of information, about surgery, by the pregnant woman and the obstetrician interest by performing such a procedure. The studies analyzed suggest also that actions to encourage normal parturition, as: the pain and suffering demystification, benefits disclosure and remuneration and assistance better for the normal parturition follow-up may reverse the current Brazilian situation
\end{abstract}

Keywords: cesarean section. incidence. pregnant women. normal birth 


\section{INTRODUÇÃO}

A evolução do nascimento de uma criança por parto normal sempre foi compreendida como um evento tradicional e natural para as mulheres. O progresso nas técnicas cirúrgicas e anestésicas em conjunto com a antibioticoterapia tornou o parto cesariano seguro e usual, estabelecendo sua utilização em larga escala (VELHO et al., 2014; WEIDLE et al., 2014). Entende-se que a decisão pelo tipo de parto compreende todo um processo físico e psicológico na gravidez e que fatores socioculturais e alusivos ao medo de parto são determinantes (OLIVEIRA; PENNA, 2018).

A cirurgia cesariana surgiu como uma alternativa ao parto normal, nos casos em que a vida da gestante, do feto, ou ambas, estivessem em risco. Este recurso deveria ser indicado pelo obstetra após avaliação das condições e circunstâncias da gestação e deve ser, portanto, realizado em casos de necessidade justificada, tendo em vista os riscos decorrentes de qualquer procedimento cirúrgico (FEBRASGO, 2018). O Guia dos Direitos da Infância e do Bebê, redigido pelo Fundo das Nações Unidas para a Infância, estabelece que a cesárea somente deve ser considerada, após o início do trabalho de parto e em situações na qual o nascimento natural prejudique a saúde ou coloque em risco a vida dos envolvidos (ANJOS et al., 2014).

Pesquisas na área de saúde apontam que, atualmente, há um uso indiscriminado deste procedimento, expondo a mulher e o bebê, a possíveis desfechos adversos. Além do aumento do risco de morbidade e dos problemas respiratórios para o bebê ou, ainda, o retardo da descida do leite materno, há também as complicações intrínsecas associadas a qualquer cirurgia, como hemorragia, infecção, entre outras. No Brasil, a cesárea está ligada a uma maior morbimortalidade materna e infantil, se comparada ao parto normal, e apesar disso, o país tem se destacado como um dos líderes na utilização deste procedimento, o que se convencionou chamar de cultura da cesariana (FAÚNDES; CECATTI, 1991; BELIZÁN et al., 2006; GIBBONS et al., 2010).

Os aspectos, como a angústia respiratória para o bebê nesse procedimento, em comparação ao normal, e as questões da morbidade e mortalidade associadas às infecções puerperais são reforçadas por outros estudos (BENINCASA et al., 2012; LIMA et al., 2014). Essas pesquisas apontam, ainda, outros riscos inerentes ao procedimento, dentre os quais: o 
risco de um erro de cálculo do tempo de gestação, em cesáreas com data agendada, que pode ocasionar uma interrupção prematura da gravidez, além das complicações e acidentes decorrentes da anestesia, como a inalação de vômito (FAÚNDES; CECATTI, 1991; HEINZMANN et al., 2009; FARCHI et al., 2010).

Segundo os dados do Sistema de Informações sobre Nascidos Vivos (Sinasc), referentes aos nascimentos em 2016, 55,4\% dos partos no Brasil foram cirúrgicos, apesar de 15\% ser considerado como taxa máxima aceitável pela Organização Mundial de Saúde. Entre os estados com maiores índices, destacam-se: Goiás (67\%), Espírito Santo (67\%), Rondônia (66\%), Paraná (63\%) e Rio Grande do Sul (63\%) (AGÊNCIA SENADO, 2016). O estudo de BARBOSA et al., (2003) reforça a ideia de que essas taxas são menores em regiões mais pobres, como as regiões Nordeste (20\%) e a Norte (25\%), e que a maioria dos procedimentos cesáreos brasileiros são realizados por mulheres com maior nível de escolaridade (BARBOSA et al., 2003). Esses achados corroboram com outro estudo que mostra o maior índice de cirurgias cesarianas entre mulheres brancas, com melhores condições socioeconômicas e que realizam seus partos em hospitais particulares. Os autores acreditam ser paradoxal compreender que as mais elevadas taxas de cesárea estejam presentes exatamente nos grupos cujas condições de saúde materna sejam melhores e risco obstétrico menor. Tal constatação sugere "um abuso da tecnologia médica no atendimento ao parto" (FREITAS et al., 2015).

A pesquisa se justifica, portanto, pela importância da reflexão a respeito do número excessivo de cesarianas realizadas no Brasil, visto que o parto normal, em condições saudáveis de gestação, é a melhor alternativa para o nascimento de uma criança. É necessário, ainda, compreender as razões que motivam as mulheres e os médicos brasileiros a optarem por esse procedimento, mesmo nos casos nos quais não há risco a vida da gestante e do bebê; e, então, sensibilizar os diversos profissionais e demais envolvidos no processo de gestação para que possam desconstruir essa "cultura da cesariana" no Brasil.

Tomando como base os dados acima, constitui objetivo geral deste estudo analisar as razões que justificam o alto índice de partos cesarianos no Brasil. São, ainda, objetivos específicos deste artigo: compreender o envolvimento do profissional como influenciador para a escolha do tipo de parto; entender o papel da mulher e os estudos feministas na tomada de decisão a respeito do procedimento e apontar alternativas de sensibilização que visem a redução da incidência de cesarianas no Brasil. 


\section{METODOLOGIA}

Este estudo se classifica como uma revisão bibliográfica, cuja fundamentação teórica foi realizada utilizando as bases de dados Scielo e Lilacs. A busca ocorreu entre agosto de 2018 e dezembro de 2019 e teve como critérios de inclusão artigos na língua portuguesa e inglesa, disponíveis online e em texto completo. Foram utilizados os descritores: parto, cesárea, incidência e gestantes. Esse levantamento reuniu 35 artigos, e após organização dos dados, como base nos descritores mais frequentes e adequados ao tema, e leitura dos resumos, foram selecionados 31 periódicos identificados como mais pertinentes para elucidação dos objetivos propostos. A inclusão dos periódicos foi realizada considerando dois aspectos principais: os fatores mais relevantes na escolha pelo tipo de parto e a importância da informação para uma decisão consciente.

\section{DESENVOLVIMENTO}

As diversas hipóteses apontadas para compreender as razões pelas quais as mulheres e os médicos optam pelo parto cesariano são baseadas, principalmente, em evidências indiretas, bem como sofrem influência de fatores socioculturais, institucionais e legais (CAMPANA; PELLOSO, 2007). Uma delas é a de que parte dos médicos tem a convicção de que o parto cesariano é mais se $\neg$ guro para o bebê e também mais confortável para a mãe. A outra é de que os profissionais em formação não foram treinados de forma eficiente para prestar assistência ao parto normal e a cirurgia cesariana seria uma forma de evitar situações arriscadas, nos quais o obstetra teria dificuldade em contornar. Por fim, o menor tempo gasto na cesariana e a possibilidade de agendamento constituem outros motivos para tomada de decisão (SASS; HWANG, 2009).

$\mathrm{Na}$ tentativa de compreender a preferência pela cesariana, outras razões são apontados como determinantes, dentre as quais: não sofrer a dor do parto, não se atentando à "possibilidade de re-ceber cuidados para alívio da dor também no parto normal, ou seja, métodos farmacológicos e não farmacológicos" (ALMEIDA et al., 2015); preocupação com a estética atrelada à crença de que o parto cesariano preserva a anatomia vaginal; e por fim, a de 
que o parto normal é mais arriscado que a cesariana, o que não é comprovado cientificamente (OLIVEIRA et al., 2016).

A preferência pela via cesariana, ainda no pré-natal, teve como fatores determinantes a idade avançada das mulheres, bem como o desejo em realizar a laqueadura. Porém, durante a realização do estudo foram destacadas, ainda, razões, como: "o medo da dor e o tempo prolongado do processo de parturição por via vaginal, a desinformação, a praticidade, a indicação do procedimento pelo médico para segurança do filho e o poder de intervenção desse profissional” (COPELLI et al., 2015). As mulheres relataram, ainda, que o médico responsável pelo acompanhamento da gestação exerce influência decisiva na escolha do tipo de parto (COPELLI et al., 2015).

Tendo em vista as razões para a escolha da cesariana ao invés do parto normal, conforme elucidado anteriormente, esta pesquisa buscou, ainda, compreender a influência do obstetra sobre a decisão da mulher em relação ao tipo de parto escolhido. Um estudo realizado com 85 gestantes demonstrou que $75,3 \%$ das mulheres negaram ter sofrido influência do obstetra sobre o tipo de parto escolhido e $24,7 \%$ confirmaram que a opinião dos profissionais influenciou na escolha (JUNIOR et al., 2013). O estudo ainda abordou as preferências dos obstetras em relação ao tipo de parto. Entre os 11 entrevistados, 63,6\% declarou preferir o parto cesariano, mas quando questionados sobre qual tipo de parto indicariam para suas esposas ou para si mesmas (no caso de profissionais do sexo feminino), 100\% responderam que seria o parto normal (JUNIOR et al., 2013).

KOTTWITZ et al., 2018 atentaram para o fato de que

poucas mulheres mencionaram terem participado da decisão do seu tipo
de parto, fato também observado na prática profissional, no qual, muitas
vezes, há um direcionamento da mulher na decisão sobre o desfecho da
gestação. Dependendo da equipe que presta a assistência durante o
trabalho de parto e do conhecimento da mulher, as opiniões dos
profissionais de saúde exercem forte influência nas decisões da
parturiente e da sua família. Observa-se ainda que, o fato da mulher não
possuir conhecimento adequado sobre a fisiologia e o tempo de trabalho
de parto, a torna mais vulnerável às decisões das equipes de saúde e reduz
seu poder de escolha (KOTTWITZ et al., 2018).

WEIDLE et al., 2014 demonstraram que, $43 \%$ das mulheres entrevistadas escolheram o parto normal por influência dos familiares e $31 \%$ foram influenciadas, pelo médico, a optar pelo parto cesariana. Também foi ressaltada que uma das razões da excessiva taxa de cesariana, 
conforme o estudo em questão é "a relação médico-paciente assimétrica, que dificulta a participação das mulheres na decisão do tipo de parto"( WEIDLE et al., 2014 ).

A influência do obstetra na decisão pelo parto cesariana é conhecida e preconizada por "um estilo de pensamento médico obstetra materializado em um conjunto de práticas, técnicas, tecnologias e saberes que cria disposições para o entendimento da cesariana como um modo normal de nascer" (NAKANO et al., 2017). O protagonismo médico decorrente de uma cesariana vem motivando gerações de profissionais a indicação dessa via de parto, fortalecendo a necessidade de fornecer, cada vez mais, alternativas ao parto normal, considerado doloroso e sofrido pela maior parte das mulheres.

Aproximadamente $88 \%$ dos recém-nascidos em hospitais privados vêm ao mundo via parto cesariana, e esses procedimentos programados e sem indicação clínica chegam a quase $25 \%$ dos partos cirúrgicos. Esse dado, somado à posição do Brasil entre os países com as taxas mais elevadas de cesarianas.

$$
\begin{aligned}
& \text { é reflexo do modelo intervencionista, no qual o processo de } \\
& \text { medicalização do corpo feminino leva à banalização da parturição } \\
& \text { cirúrgica e o pouco conhecimento da população sobre a autonomia da } \\
& \text { parturiente no processo de decisão da via de parto intensifica esse fato } \\
& \text { insustentável (PIMENTEL;OLIVEIRA-FILHO, 2016). }
\end{aligned}
$$

De acordo com os dados da Associação Médica Brasileira, a remuneração de um parto cesáreo é um pouco maior do que de um parto normal, em hospitais privados. No entanto, esses custos podem, no caso de procedimento cirúrgico, alcançar valores exorbitantes, considerando o obstetra, um auxiliar e/ou instrumentador, um anestesista e um pediatra neonatal. Além disso, o fator tempo também é relevante, visto que a duração de uma cesariana é de aproximadamente 3 horas, enquanto o acompanhamento de um parto normal, dependendo do caso, pode ultrapassar 12 horas. Esses fatores demonstram que há um interesse, dos hospitais e até dos próprios profissionais envolvidos no processo de gestação, pela realização do parto cesariana, no qual o agendamento prévio das cesarianas maximiza o uso dos leitos e remunera melhor os obstetras.

O parto cesariano ganhou, portanto, um "outro caráter além do de procedimento médico: o de atividade lucrativa". A cirurgia cesariana se tornou um bem de consumo, acessível para gestantes e famílias com maior poder aquisitivo. 
Esta constatação não toma como base apenas os valores cobrados pelos tipos de parto, mas também as questões do agendamento e do tempo de assistência dedicado ao parto cesariana. Esses privilégios que o parto cesariano possibilita aos médicos, alinhados aos benefícios para as gestantes (agendamento do parto, redução da dor, transferência do trabalho de parto para o obstetra), fizeram com que o parto cesariano conquistasse mais adeptos, tanto profissionais da área como mulheres em geral, ao longo dos últimos anos (BARCELLOS et al., 2009).

Além disso, é notória a constituição do médico como formador de opinião, de forma que a escolha pela via de parto é consequência, em parte, de como as gestantes são informadas sobre os riscos e benefícios das alternativas para o nascimento do bebê (PIMENTEL; OLIVEIRA-FILHO, 2016).

Acredita-se que o imaginário popular acerca das vias de parto e seus impactos sobre a vida e o corpo da mulher podem explicar essa cultura da cesariana no Brasil (FREIRE et al., 2011). De acordo com o estudo,

o suporte familiar e o tipo de atividade laboral exercem influência sobre a escolha, além de nível econômico, acesso ao serviço de saúde, à educação e informação e aos fatores culturais, que também interferem, ou mesmo determinam afinidade da mulher em relação ao parto normal e cesariana (FREIRE et al., 2011).

Foram pontuadas que a escolha pela via de parto pela mulher ocorre no decorrer da gestação e, para a tomada de decisão, elas demandam um atendimento individual, no qual possam obter informações verídicas. De acordo com o estudo, "essa falta de informação contribui para o aparecimento de dúvidas e questionamentos em relação a qual tipo de parto vivenciar após uma cesárea anterior". O estudo sugere, ainda, que a representação feminina no processo de parturição tem sido simbolizada pelas apreensões e conflitos de sentimentos gerados pelo medo da dor, e que essa visão tem sido reforçada pelo modelo biomédico e pela mídia (ESCOBAL et al., 2018).

Foi realizada uma importante reflexão acerca das mudanças no processo de parturição ao longo dos anos,

e a partir do século XX na década de 40, foi intensificada a hospitalização do parto, que permitiu a medicalização e controle do período gravídico puerperal e o parto como um processo natural, privativo e familiar, passou a ser vivenciado na esfera pública, em instituições de saúde com a presença de vários atores conduzindo este período. Esse fato favoreceu 
a submissão da mulher que deixou de ser protagonista do processo parturitivo (MOURA et al., 2007).

O parto, que antes acontecia dentro das casas das gestantes, se tornou uma prática intervencionista, sem o protagonismo e o direito de escolha da mulher (RISCADO et al., 2016). Tais acontecimentos resultaram, ao longo dos anos, em um despreparo das mulheres perante o parto normal, "interferindo diretamente no sistema emocional da gestante ou parturiente, diminuindo a confiança dela na capacidade de ser protagonista do seu parto" (WEIDLE et al., 2014). O momento do parto deve reafirmar a competência da mulher. No entanto, o medo do sofrimento pode torná-la incapaz de compreender os benefícios do parto normal, sendo, então, conduzida a escolher a cesariana, como se esta fosse a melhor opção para si e para o seu bebê. Os movimentos feministas defendem que as mulheres têm total autonomia e poder de decisão para a realização do parto cesariano ou do parto normal, sendo essencial o atendimento dos seus direitos, assim como o fornecimento de informações sobre os benefícios e malefícios da sua escolha.

Nesta perspectiva, uma primeira alternativa de sensibilização para a redução de cesarianas no Brasil seria a revisão dos valores de repasse pela execução de cada um destes procedimentos, valorizando mais o acompanhamento médico de um parto normal do que o parto cesariano (apesar deste demandar maior técnica). Juntamente com essa medida, também é grande importância que o Ministério da Saúde divulgue, em maior escala, os benefícios do parto normal, de forma a incentivar as mulheres a optarem por esta via.

Esta sensibilização pode ser feita por meio de campanhas nacionais nos meios de comunicação de massa (TV, rádio e redes sociais), e por meio de ações e eventos nas comunidades. Felizmente, os benefícios do parto normal têm se popularizado entre grupos de mulheres e grupos feministas, que tem se engajado em ações favoráveis ao chamado parto humanizado. A expectativa é de que as futuras gestantes tenham esse conhecimento e possam, nos próximos anos, reverter o atual quadro brasileiro de cultura da cesariana.

A implementação de uma rotina de acompanhamento para gestantes, que realizaram uma cesariana previamente, deve ser proposta, a fim de romper com o 
ciclo de repetição e reduzir o índice de cesáreas por esta indicação. "Isso requer uma melhor definição dos critérios na triagem e acompanhamento adequado do processo do trabalho de parto" (FAÚNDES; CECATTI., 1991). Outras medidas para a redução do número de partos cesarianos, são: o uso de partograma; capacitação da equipe de profissionais que proporciona assistência às gestantes; e resgate da atuação da parteira por meio de um pré-natal que prepare a mãe, física e psicologicamente, para o trabalho de parto (FAÚNDES; CECATTI., 1991).

A humanização da assistência à saúde é premissa do SUS e das instituições da área. No entanto, a humanização do parto e a assistência desse tipo de procedimento, do modo como é conduzido pelo sistema público de saúde, limita a sua utilização, sendo realizado, muitas vezes, apenas pelas mulheres que não tem outra alternativa. Em uma perspectiva de realização adequada, o serviço permitiria mais qualidade e segurança à mulher e ao seu bebê. Ao mesmo tempo, a gestante poderia atuar como protagonista, "fazendo com que o parto deixasse de ser um evento meramente biológico, e sim uma experiência humana, que deve ser experimentada de acordo com suas expectativas" (DIAS; DOMINGUES, 2005).

Nesse sentido, são apontadas nove estratégias para a redução de partos cesarianos desnecessárias no país. São elas: I- definir as indicações precisas para a realização de uma cesárea; II- utilizar protocolos e fluxogramas baseados em evidência, elaborado por especialistas; III- solicitar uma segunda opinião médica antes da realização da cesárea; IV-concentrar os esforços de sensibilização no grupo de mulheres que estão em sua primeira gestação, para evitar o efeito da primeira cesárea; V- recomendar a indução de parto, explicando seus benefícios; VI- realizar partogramas e melhorar a experiência do parto normal para a mulher; VII- conhecer e adotar práticas que tornem o trabalho de parto, bem como o parto mais seguro, evitando intervenções desnecessárias; VIII- utilizar alternativas de combate à dor, promovendo mais satisfação para a mulher durante o parto; IX- realizar a monitorização do feto da gestação ao parto; e, consequentemente, proporcionar todos os procedimentos especiais, com a presença de profissionais especialistas exigidos pela condição do feto e da gravidez durante o parto (HADDAD; CECAT, 2011). 


\section{CONSIDERAÇÕES FINAIS}

Concluiu-se que a falta de conhecimento, o medo da dor, o mito de que a cesariana é mais segura para o feto, a precária assistência concedida à mulher durante o trabalho de parto, além das outras razões apontadas no desenvolvimento deste artigo são determinantes na escolha do parto e podem ajudar a explicar o fenômeno denominado cultura da cesariana no Brasil. Notouse também que o tempo é um fator encorajador da preferência pela via de parto cesariana, uma vez que os obstetras, que indicam essa prática, têm a possibilidade de agendamento e, por outro lado, a gestante evita um processo que pode ser prolongado e, com a falta de amparo profissional, doloroso e difícil.

Quanto aos fatores que influenciam na escolha da mulher pelo parto cesariano, percebeuse que os obstetras e familiares da gestante tem papel importante. Esta constatação deve ser trabalhada em próximos estudos, uma vez que aponta a demanda de uma desmistificação dos prejuízos do parto normal, além de um trabalho de conscientização e capacitação de profissionais ligados à área, tornando-os potenciais incentivadores do parto normal. Colocar a mulher no papel central, dando a ela o poder de decisão e oferecendo-a escolhas, torna o processo mais prazeroso e tranquilo.

O parto humanizado, encorajado por feministas e profissionais adeptos, tem ganhado visibilidade. Atualmente é possível encontrar clínicas especializadas neste atendimento, nas quais o acompanhando da mulher, durante a gestação e o parto, está direcionado para o bem estar da mesma, permitido uma melhor experiência no parto normal. Essa prática precisa se fortalecer e efetivamente ser aplicada nos hospitais particulares e Sistema Único de Saúde (SUS), garantido à mulher uma escolha consciente, segura e positiva.

\section{REFERÊNCIAS}

AGÊNCIA SENADO., 2016. Disponível em: https://www12.senado.leg.br/noticias/especiais/especialcidadania/especialistas-apontamepidemia-de-cesarianas/especialistasapontamepidemia-de-cesarianas. Acesso em: 19 de dezembro de 2019. 
ALMEIDA, J. M.; ACOSTA, L. G.; PINHAL, M. G. Conhecimento das puérperas com relação aos métodos não farmacológicos de alívio da dor do parto. Rev Min Enferm. 2015:19(3): 718-724.

ANJOS, C. S.; WESTPHAL, F.; GOLDMAN, R. E. Cesárea Desnecessária no Brasil: Revisão integrativa. Enferm. Obstétrica, Rio de Janeiro, 2014: 1(3):86-94.

BARBOSA, G. P.; GIFFIN, K.; ANGULO-TUESTA, A.; GAMA, A. S.; CHOR, D.; DORSI, E.; REIS, A. C. G. V. Parto cesáreo: quem o deseja? Em quais circunstâncias? Cad. Saúde Pública. 2003: 19 (6): 1611-1620.

BARCELLOS, LG.; SOUZA, A. O. R.; MACHADO, C. A. F. Cesariana: uma visão bioética. Rev. Bioética. 2009: 17(3): $497-510$.

BELIZÁN, J. M.; CAFFERATA, M. L.; ALTHABE, F.; BUEKENS, P. Risk of patient choice cesarean. Birth. 2006: 33(2):167-9.

BENINCASA, B. C.; WALKER, C.; CIOBA, C.; ROSA, C. C. S.; MARTINS, D. E.; DIAS, E.; KLUCK, M. Taxas de infecção relacionadas a partos cesáreos e normais no Hospital de Clínicas de Porto Alegre. Rev HCPA 2012:32(1).

CAMPANA, H. C. R.; PELLOSO, S. M. Levantamento dos partos cesárea realizados em um hospital universitário. Rev. Eletr. Enferm. 2007: 9(1):51-63.

COPELLI, F. H. S.; ROCHA, L.; ZAMPIERI, M. F. M.; GREGÓRIO, V. R. P.; CUSTÓDIO, Z. A. O. Fatores determinantes para a preferência da mulher pela cesariana. Texto Contexto Enferm. 2015: 24 (2): 336-43.

DIAS, M. A. B.; DOMINGUES, R. M. S. M. Desafios na implantação de uma política de humanização da assistência hospitalar ao parto. Cien. Saúde Colet. 2005: 10 (3) - 669 - 705.

ESCOBAL, A. P.; MATOS, G. C.; GONCALVES, K. D.; QUADRO, P. P.; CEGAGNO, S.; SOARES, M.C. Participação da mulher na tomada de decisão no processo de parturição. Rev. Enferm. 2018: 12(2):499-509.

FARCHI, S.; POLO, A.; FRANCO, F.; DI LALLO, D.; GUASTICCHI, G. Severe postpartum morbidity and mode of delivery: a retrospective cohort study. Acta Obstet Gynecol Scand. 2010: 89(12):1600-3.

FAÚNDES, A.; CECATTI, J. C. A operação Cesárea no Brasil. Incidência, tendências, causas, consequiências e propostas de ação. Rio de Janeiro, 1991:7 (2): 150-173.

FEBRASGO, 2018. Disponível em: https://www.febrasgo.org.br/pt/noticias/item/728-altataxa-de-cesareas-o-brasil-e-tema-de-audiencia-publica. Acesso em: 19 de dezembro de 2019.

FREIRE, N. C.; NUNES, I. M.; ALMEIDA, M. S.; GRAMACHO, R. C. C. V. Parto normal ou cesárea? A decisão na voz das mulheres. Rev. Baiana de Enferm.Salvador, 2011: 25(3): 237-247. 
FREITAS, P. F.; MOREIRA, B. C.; MANOEL, A. L.; BOTURA, A. C. A. O parecer do Conselho Federal de Medicina, o incentivo à remuneração ao parto e as taxas de cesariana no Brasil. Rio de Janeiro, 2015: 31(9):1839-1855.

GIBBONS, L.; BELIZÁN, J. M.; LAUER, J. Á.; BETRÁN, A. P.; MERIALDI, M.; ALTHABE, F. The global numbers and costs of additionally needed and unnecessary caesarean sections performed per year: overuse as a barrier to universal coverage. Geneva; 2010 World Health Report Background Paper. Disponível em: http://www.who.int/healthsystems/topics/financing/ healthreport/30C-sectioncosts.pdf.

HEINZMANN, A.; BRUGGER, M.; ENGELS, C.; PRÖMPELER, H.; SUPERTI-FURGA, A.; STRAUCH, K.; KRUEGER, M. Risk factors of neonatal respiratory distress following vaginal delivery and caesarean section in the German population. Acta Pediatrica, 2009: 98(1), 25-30.

HADDAD, S. E. M. T.; CECAT, J. G. Estratégias dirigidas aos profissionais para a redução das cesáreas desnecessárias no Brasil. Rev. Bras. Ginecol Obstet. 2011: 33(5):252-62.

JUNIOR, T.L.; STEFFANI, J. Á.; BONAMIGO, E. L. Escolha da via de parto: expectativa de gestantes e obstetras. Rev. bioét. (Impr.). 2013: 21 (3): 509-17.

KOTTWITZ, F.; GOUVEIA, H. G.; GONCALVES, A. C. Via de parto preferida por puérperas e suas motivações.Porto Alegre, RS. 2018: 22(1):e20170013.

LIMA, D. M.; WALL, M. L.; HEY, A.; FALCADE, A. C.; CHAVES, A. C. M.; SOUZA, M. A. R. Fatores de riscos para infecção no puerpério cirúrgico. Cogitare Enferm. 2014 Out/Dez: 19(4):734-40.

MOURA, F. M. J.; CRIZOSTOMO, C. D.; MENDONCA, R. C. M.; ARAÚJO, O. D.; ROCHA, S.S. A humanização e a assistência de enfermagem ao parto normal. Rev. Bras. Enferm., Brasília 2007: 60(4):452-5.

NAKANO, A.R.; BONAN, C.; TEIXEIRA, L.A. O trabalho de parto do obstetra: estilo de pensamento e normalização do "parto cesáreo" entre obstetras. Physis. Rev. de Saúde Coletiva 2017: 27 (3): 415-432.

OLIVEIRA, R. R.; MELO, E. C.; NOVAES, E. S.; FERRACIOLI, P. L. R. V.; MATHIAS, T. A. F. Fatores associados ao parto cesárea nos sistemas público e privado de atenção à saúde. Ver. Esc. Enferm. USP. 2016: 50(5):733-740.

OLIVEIRA, V. J.; PENNA, C. M. M. Cada parto é uma história: processo de escolha da via de parto. Rev. Bras. Enferm. 2018: 71(3): 1228-1236.

PIMENTEL, T. A.; OLIVEIRA-FILHO, E. C. Fatores que influenciam na escolha da via de parto cirúrgica: uma revisão bibliográfica. Universitas: Ciên. da Saúde 2016: 14 (2): 187 199.

RISCADO, L. C.; JANNOTTI, C. B.; BARBOSA, R. H. S. A decisão pela via de parto no brasil: temas e tendências na produção da saúde coletiva. Texto Contexto Enferm, 2016: 25(1):e3570014. 
SASS, N.; HWANG, S.M. Dados epidemiológicos, evidências e reflexões sobre a indicação de cesariana no Brasil. Diagn Tratamento 2009: 14(4):133-7.

VELHO, M. B.; DOS SANTOS, E. K. A.; COLLAÇO, V. S. Parto normal e cesárea: representações sociais de mulheres que os vivenciaram. Rev. Bras. Enferm.2014:67(2):282-9

WEIDLE, W. G.; MEDEIROS, C. R. G.; GRAVE, M. T. Q.; BOSCO, S. M. Escolha da via de parto pela mulher: autonomia ou indução? Cad. Saúde Colet., 2014: 22 (1): 46-53. 